\title{
Benefits of Exercise for the Prevention of Diseases in the Premature Aging
}

Robert Lugo ${ }^{1}$, Carmel B. Dyer ${ }^{2}$ and Yong $\mathrm{Li}^{* 3}$

${ }^{1}$ Department of Physical Medicine and Rehabilitation, Baylor College of Medicine, Houston, USA

${ }^{2}$ Department of Internal Medicine, University of Texas Medical School at Houston, Houston, USA

${ }^{3}$ Center for Stem Cell and Regenerative Medicine, Brown Foundation Institute of Molecular Medicine, University of Texas Health Science Center, Houston, USA

"Corresponding author: Yong Li, Center for Stem Cell and Regenerative Medicine, Brown Foundation Institute of Molecular Medicine, University of Texas Health Science Center, Houston, USA, Tel: 713-500-2438; E mail: Yong.Li.1@uth.tmc.edu

Received date: June 06, 2016, Accepted date: June 22, 2016, Published date: June 29, 2016

Copyright: (c) 2016 Lugo R, et al. This is an open-access article distributed under the terms of the Creative Commons Attribution License, which permits unrestricted use, distribution, and reproduction in any medium, provided the original author and source are credited.

\begin{abstract}
We live in a time where there is a projected increase in the number of adults surviving to advanced age. With that comes a tremendous financial burden in caring for these elderly individuals, who are likely to develop chronic diseases; both cardiovascular and neurological. Multiple studies have clearly demonstrated the benefits of exercise for preventing cardiovascular disease and age-related cognitive declines. Given the foreseeable human and economic challenges in caring for a vast elderly population with chronic disease, it is clear that appropriate exercise prescriptions could be a feasible, cost effective and therapeutic intervention measure for preventing cardiovascular and neurological diseases. However, not all older patients may possess the physical or cognitive capacity to perform physical exercise. Therefore, delineating the pathways that mediate these exercise-induced benefits and understanding how to manipulate them in vivo, may yield novel therapeutic approaches to aging prevention.
\end{abstract}

Keywords: Exercise; Cardiovascular disease prevention; Aging prevention; Neurogenesis

\section{Introduction}

As evidence by empirical observation as well as objective data, it is apparent that regular exercise leads to improvements in muscle strength, reduced risk of cardiovascular diseases, and promotes the prevention of premature aging. Some examples of cardiovascular diseases include coronary heart disease, hypertension, and stroke; while age-related decline often affects the neurological system, leading to dementia and balance prohibition among other neurocognitive impairments.

In general, there are two forms of exercise: Anaerobic and aerobic. Anaerobic exercise encompasses physical activities that occur in less than 10 seconds, utilizing glycolysis for energy needs. In addition to others, examples of anaerobic exercise include strength training, sprinting, and jump training. Aerobic exercise occurs with activity lasting greater than 10 seconds, utilizing macronutrients as substrate for energy needs. Some examples of aerobic exercise include walking, running, swimming, and bicycling.

It is becoming common knowledge that regular exercise reduces the risk of cardiovascular diseases by decreasing blood pressure, reducing truncal obesity, increasing insulin sensitivity, and improving cholesterol profile. Older individuals who continue to regularly perform exercise are able to respond to stimuli quicker and score higher during standardized cognitive tests compared to their nonexerciser counterparts [1]. Thus, regularly scheduled exercise is highly recommended, especially to elder populations.

\section{Exercise and Cardiovascular Disease Prevention}

According to the World Health Organization (WHO) in 2012, coronary heart disease (CHD) was the number one cause of death worldwide, followed by stroke at number two [1]. However, investigations indicated that frequent aerobic exercise results in numerous cardiovascular benefits, leading to a potentially reduced death rate. Using an epidemiologic measure, the population attributable fraction (PAF), researchers were able to estimate the proportion of new cases of non-communicable diseases, including $\mathrm{CHD}$ that would not occur if the risk factor of physical inactivity were eliminated. According to the PAF, they estimated that physical inactivity was responsible for $6 \%$ of the burden from CHD worldwide [2], indicating the significance for a regularly scheduled exercise prescription in the elderly population.

A meta-epidemiologic study that included 305 randomized controlled trials found there is no statistical difference between exercise and pharmacologic therapy in the secondary prevention of $\mathrm{CHD}$; however, frequent exercises is able to be more beneficial than pharmacologic therapy among patients with stroke [3]. Since 1991, the World Bank and the WHO have continued to assess the global burden of disease (GBD). As warning, physical inactivity was ranked 10th as an attributable risk factor for GBD in 2010 [4]. An observational cohort study between 1970 and 1989 found low fitness to be an independent predictor of mortality in United States residents [5]. There is a prospective cohort study that assessed the health benefits of different volumes of physical activity in a Taiwanese population between 1996 and 2008, which also found that an additional 15 minutes of daily exercise prescription beyond the minimum amount of 15 minutes per day further reduced all-cause mortality in a dosedependent manner [6]. Thus, several benefits of exercise for cardiovascular disease prevention have been suggested.

\section{Potential Mechanisms of Exercise and Cardiovascular Disease Prevention}

Exercise is ubiquitously known to prevent and treat cardiovascular diseases by reducing cardiovascular risk factors, such as diabetes 
Page 2 of 4

mellitus and hypertension; however, the mechanisms behind the molecular signaling involved with these beneficial effects are still under investigation. The benefits of exercise are systemic and a number of studies have analyzed potential mechanism at the level of the myocardium [7]. There are a multitude of cellular and molecular mechanisms involved in the cardiac response to exercise, including cardiomyocyte hypertrophic remodeling and renewal, improved cardiomyocyte calcium sensitivity and contractility, vascular endothelial growth factor (VEFG)-related remodeling, and alterations in metabolism via mitochondrial biogenesis (Table 1) [8].

The heart has the capacity for myocardium hypertrophy which occurs in response to both pathologic and physiologic stimuli. Examples of pathological and physiological stimuli include chronic hypertension and exercise, respectively. The prototypical example of physiological cardiac growth occurs in response to exercise. In contrast to pathological-induced cardiomyocyte hypertrophy, both systolic and diastolic function are preserved and possibly improved, in exerciseinduced hypertrophy [9]. Physiologic cardiomyocyte remodeling is promoted by aerobic exercise training. Fernandes et al. [10] reviewed the effects of both swimming and running on cardiac specific microRNA (miRNA) expression and concluded that physiologic remodeling was regulated by the effect of these small ( 22 nucleotides in length), endogenous, non-encoding RNAs on target genes. Consistent with the role of miRNAs, Chen et al. [11] demonstrated that cardiac-specific knockout Dicer, a gene encoding a RNase III endonuclease essential for miRNA processing, leads to rapidly progressive dilated cardiomyopathy and heart failure in mice. Exercise training has been shown to promoted increased expression of miRNA-126, leading to both angiogenesis and vasodilation via the VEGF pathway [12], and subsequent enhanced oxygen delivery to the myocardium. Zhou et al. [13] analyzed VEGF concentration, blood vessel density, and mRNA expression of VEGF in rats that were randomly assigned to two groups: low-intensity treadmill exercise versus non-exercise. They concluded that VEGF concentration, blood vessel density, and mRNA expression of VEGF was greater in the exercise group than in the non-exercise group. Conclusively, the evidence in the literature demonstrates the important role of miRNA- and VEGF-mediated cardiovascular remodeling as a result of exercise.

\begin{tabular}{|l|}
\hline \multicolumn{1}{|c|}{ Improvement in: Heart } \\
\hline Cardiomyocyte hypertrophic remodeling \& renewal \\
\hline Cardiomyocyte calcium sensitivity \& contractility \\
\hline Vascular endothelial growth factor (VEGF)-related remodeling \\
\hline Metabolism via mitochondrial biogenesis \\
\hline
\end{tabular}

Table 1: Cardiac benefits of aerobic exercise

Researchers have shown that the regenerative potential of the heart is dynamically regulated, and that endurance exercise may promote existing cardiomyocyte or progenitor cell proliferation and differentiation in the resident heart, which resembles that of the hippocampal neurogenesis that has been observed in response to aerobic exercise $[14,15]$.

\section{Exercise and Aging Prevention}

As the brain ages there is a decline in motor and cognitive processes that is part of normal aging; however, with the implementation of aerobic exercise, such as walking, running, swimming, and bicycling, these age-related deficits have been shown to be preventable (Table 2). In general, the effect of exercise on the cognitive function of older individuals appears to be systemically beneficial. For individuals who have participated in aerobic exercise for the majority of their lives, they have been explored to outperform non-exercisers in stimuli discrimination, response to audio or visual stimuli, and various standardized tests [9]. A prospective study of nearly six-thousand elderly females discovered that women with higher physical activity tolerance levels at baseline were not as likely to experience cognitive decline [10].

It has been demonstrated that even previously healthy-sedentary adults between the ages of 60-75 can significantly improve their reaction time performance, their ability to ignore irrelevant visual information as well as to coordinate multiple tasks, after 6-months of aerobic exercise intervention [11]. A meta-analysis that looked at all randomized fitness trials with control groups from 1966 to 2002 with adults over the age of 55 concluded that the impact of aerobic fitness was greater for executive control functions, such as planning, scheduling, and coordinating [12].

Normal aging results in a decrease in brain volume, particularly at the frontal lobe where higher order cognitive processes occur, such as working memory, switching between tasks, and inhibiting irrelevant information [12]. Researchers conducted a randomized controlled trial which found a significant increase in frontal lobe volume in healthy elderly individuals' between the ages of 60-79 years old after undergoing a 6-month aerobic exercise program compared to agematched controls that did not undergo aerobic exercise [16].

\begin{tabular}{|l|}
\hline \multicolumn{1}{|c|}{ Improvement in: Brain } \\
\hline Motor \& cognitive processes \\
\hline Reaction time performance \\
\hline Ability to ignore irrelevant visual information \\
\hline Ability to perform multiple tasks \\
\hline Executive functions (i.e., planning, scheduling, and coordinating) \\
\hline Memory \\
\hline Increase in: \\
\hline Hippocampus volume \\
\hline Frontal lobe volume \\
\hline Cerebral perfusion and metabolic support \\
\hline
\end{tabular}

Table 2: Neurologic benefits of aerobic exercise 


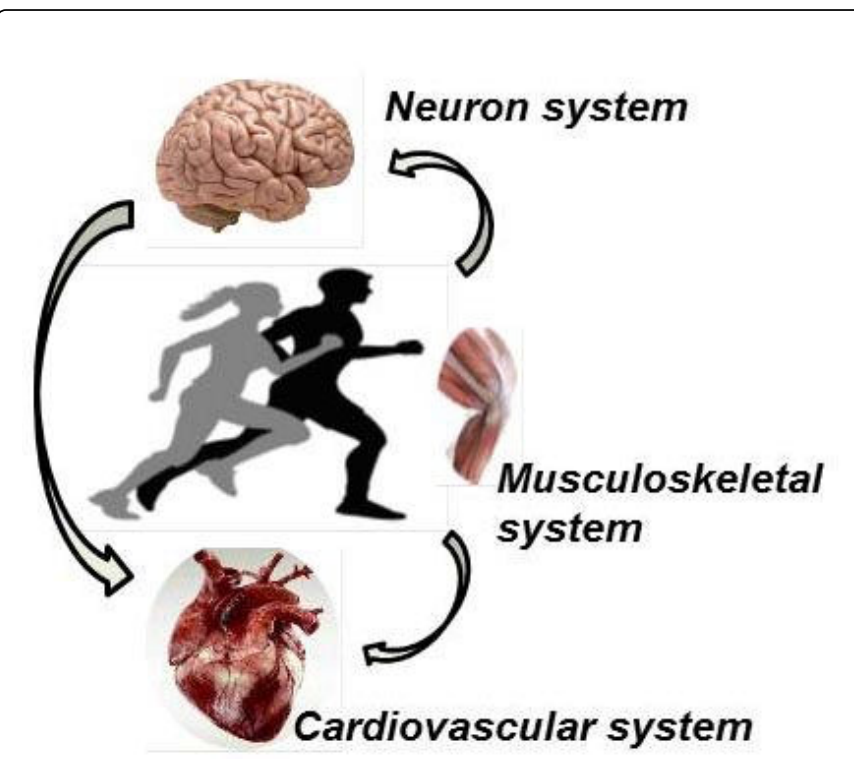

Figure 1: Exercise prescription modulates the cerebral and cardiovascular system

\section{Potential Mechanisms of Exercise and Aging Prevention}

Researchers have demonstrated improved cerebral perfusion and metabolic support in response to exercise and stimulating environments. In ponies, the oxygen demands of the cerebellum and brain stem are greater than the cerebral cortical areas when undergoing significant levels of exercise [7]. Regular exercise was shown to shorten blood vessel diffusion distance as well as promote angiogenesis and synaptogenesis in the paramedian lubule of the cerebellum in rats [8]. There was an increase in the density of the cerebral cortex neuropil that positively correlates in rats confined to stimulating compared to non-stimulating environments [13].

Similar to adult humans, their hippocampus maintains the capacity to generate neurons in general. In areas of the brain previously identified as neurogenic in adult rodents and monkeys, researches used immunofluorescent labeling to demonstrate new neurons from progenitor cells in the dentate gyrus of adult humans $[17,18]$. Neurogenesis occurs at the level of the granule cell layer of the adult dentate gyrus, which can be modulated by sex hormones and experience [19]. Age-related decreased production of new neurons in the neurogenic dentate gyrus has been associated with memory impairments [20]. The hippocampus shrinks in late adulthood, leading to impaired memory and increased risk for dementia. In a randomized controlled trial, researchers have shown that exercise increased the size of the hippocampus and improved memory of 120 adults with a mean age of 67.6 years old [21].

\section{Discussion}

The literature supports the fact that elderly individuals with higher fitness levels tend to outperform their sedentary counterparts at both physical and cognitive assessments. The outcomes of retrospective, cross-sectional, and prospective studies suggest a correlation between the cognitive function of older adults and their baseline fitness level.
Other covariates that may account for all or part of the relationship between fitness level and cognitive function include lifestyle preferences, such as diet, smoking, and nutrition.

Given what we know regarding the exercise-induced benefits of cardiovascular diseases and the prevention of cognitive decline in the elderly population, is there a way to stimulate minimal exercise in the frail or medically complicated patient, who may not be able to physically exercise, at the molecular level? Further research is needed to better understand the pathways that modulate the cardiovascular exercise-induced benefits [22]. Understanding how to manipulate these pathways in vivo, may yield novel therapeutic approaches to allow the universal prescription of cardiovascular exercise for the prevention of cardiovascular disease and premature aging.

\section{Conclusion}

In this review, we discussed the inevitable financial burden in caring for elderly individuals, who are likely to develop chronic cardiovascular and neurological diseases. The impact that cardiovascular diseases has worldwide, the effect that low fitness has on mortality, the result of cellular and molecular mechanisms involved in the cardiac response to exercise, the heart's capacity to generate new cardiomyocytes or progenitors, which resembles that of the hippocampal neurogenesis as a response to aerobic exercise. Finally, we mentioned how the aging process affects the brain over time and that with proper exercise prescription there is an increase in cerebral angiogenesis, synaptogenesis, and neurogenesis, resulting in increased volume in areas known to function in higher order processes (Figure 1) [22-26].

It is clear that appropriate exercise prescription can be a feasible and cost effective preventative and therapeutic intervention for treating cardiovascular and neurological diseases associated with aging; however, many patients may not possess the physical nor cognitive capacity to perform regular physical exercise. Therefore, delineating the pathways that mediate these exercise-induced benefits and understanding how to manipulate them in vivo, may yield novel therapeutic approaches to the prevention of disease and premature aging.

\section{References}

1. http://www.who.int/mediacentre/factsheets/fs310/en/. Accessed on April 3,2015

2. Lee (2012) Impact of Physical Inactivity on the World's Major NonCommunicable Diseases. Lancet 380: 219-229.

3. Naci H, Ioannidis J (2013) Comparative effectiveness of exercise and drug interventions on mortality outcomes: metaepidemiological study. BMJ 347: 577.

4. Murray CJ, Phil D, Lopez AD (2013) Measuring the global burden of disease. N Engl J Med 369:448-457.

5. Blair SN, Kampert JB, Kohl HW, Barlow CE, Macera CA, et al. (1996) Influcences of cardiorespiratory fitness and other precursors on cardiovascular disease and all-cause mortality in men and women. JAMA 276: 205-210.

6. Wen CP, Wai JPM, Tsai MK, Yang YC, Cheng TYD, et al. (2011) Minimum amount of physical activity for reduced mortality and extended life expectancy: a prospective cohort study. Lancet 378: 1244-1253.

7. Manohar M (1986) Regional blood flow and oxygen delivery during severe exertion in the pony. Respir Physiol 64:339-349.

8. Isaacs KR, Anderson JT, Alcantara AA, Black JE, Greenough WT (1992) Exercise and the brain: angiogenesis in the adult rat cerebellum after 
Citation: Lugo R, Dyer CB, Li Y (2016) Benefits of Exercise for the Prevention of Diseases in the Premature Aging . J Aging Sci 4: 154. doi:

vigorous physical activity and motor skill learning. J Cereb Blood Flow Metab 12: 110-119.

9. Churchill JD, Galvez R, Colcombe S, Swain RA, Kramer AF, et al. (2002) Exercise, experience and the aging brain. Neurogiol Aging 23: 941-955.

10. Yaffe K, Barnes D, Nevitt M, Lui LY, Covinsky K (2001) A prospective study of physical activity and cognitive decline in elderly women who walk. Arch Int Med 161:1703-1708.

11. Kramer AF, Hahn S, Cohen NJ, Banich MT, McAuley E, et al. (1999) Aging, fitness and neurocognitive function. Nature 400:418-419.

12. Colcombe S, Kramer AF (2003) Fitness effects on the cognitive function of older adults: a meta-analytic study. Psychological Sci 14:125-130.

13. Bennett EL, Diamond MC, Krech D, Rosenzweig MR (1964) Chemical and anatomical plasticity of brain. Science 146:610-619.

14. Eriksson PS, Perfilieva E, Bjork-Eriksson T, Alborn AM, Nordborg C, et al. (1998) Neurogenesis in the adult human hipposcampus. Nat Med 4:1313-1317.

15. Kempermann G, Gage FH (2000) Neurogenesis in the adult hippocampus. Novartis Found Symp 231:220-235.

16. Gould E, Tanapat P, Rydel T, Hastings N (2000) Regulation of hippocampal neurogenesis in adulthood. Biol Psychiatry 48:715-720.

17. Seib DR, Martin-Villalba A (2015) Neurogenesis in the normal aging hippocampus: A mini-review. Gerontology 61:327-335.

18. Bowles DK, Farrar RP, Starnes JW (1992) Exercise training improves cardiac function after ischemia in the isolated, working rat heart. Am J Physiol 263: H804-H809.
19. Mann N, Rosenzweig A (2012) Can exercise teach us how to treat heart disease? Circulation 126:2625-2635.

20. Fernandes T, Barauna VG, Negrao CE, Phillips MI, Oliveira EM (2015) Aerobic exercise training promotes physiological cardiac remodeling involing a set of microRNAs. Am J Physiol Heart Circ Physiol 309: H543H522.

21. Chen (2008) Targeted deletion of Dicer in the hearts leads to dilated cardiomyopathy and heart failure. PNAS 105: 2111-2116.

22. Zhou (2016) Low-intensity treadmill exercise promotes rat dorsal wound healing. J Hauzhong Univ Sci Technol 36: 121-126.

23. Bostrom P, Mann N, Wu J, Quintero PA, Plovie ER, et al. (2010) C/ EBPbeta controls exercise-induced cardiac growth and protects against pathological cardiac remodelin. Cell 143:1072-1083.

24. Erickson KI, Voss MW, Prakash RS, Basak C, Szabo A, et al. (2011) Exercise training increases size of hippocampus and improves memory. Proc Natl Acad Sci USA 108:3017-3022.

25. American Hospital Association (2007) First Consulting Group. When I'm 64; How boomers ill change health care. Chicago: American Hospital Association.

26. Colcombe SJ, Erickson KI, Scalf PE, Kim JS, Prakash R, et al. (2006) Aerobic exercise training increases brain volume in aging humans. J Gerontol A Biol Sci Med Sci 61: 1166-1170. 